\title{
Employing FREYA for fission product yield evaluations
}

\author{
$R$. Vogt ${ }^{1,2, *}, J$. Randrup ${ }^{3, * *}, N$. Vassh $^{4}, T$. Sprouse ${ }^{4}$, and $R$. Surman ${ }^{4}$ \\ ${ }^{1}$ Nuclear and Chemical Sciences Division, Lawrence Livermore National Laboratory, Livermore, CA \\ 94551, USA \\ ${ }^{2}$ Physics Department, University of California at Davis, Davis, CA 95616, USA \\ ${ }^{3}$ Nuclear Science Division, Lawrence Berkeley National Laboratory, Berkeley, CA 94720, USA \\ ${ }^{4}$ Department of Physics, University of Notre Dame, Notre Dame, IN 46556, USA
}

\begin{abstract}
The fast event-by-event fission code FREYA (Fission Reaction Event Yield Algorithm) generates large samples of complete fission events while employing only a few physics-based parameters. Not only is FREYA fast, it is also flexible, able to employ a variety of input formats to test the implications of various fission yield evaluations on neutron and photon observables. We describe how FREYA was applied to the neutron-rich nuclei needed for $r$-process nucleosynthesis calculations as an example of this flexibility. Finally, we discuss how we plan to make use of this flexibility to extend FREYA to calculations of cumulative fission product yields to aid evaluations of these yields in the future.
\end{abstract}

\section{Introduction to FREYA}

As input, the standard version of FREYA requires the mass distribution of the initial fission fragments, $Y(A)$, and the mean total kinetic energy for a given mass split, $\overline{\operatorname{TKE}}(A)$. These distributions taken from either experimental data or model calculations. There are a number of approaches that can be employed for such calculations, as described elsewhere in these proceedings [1-3]. They are generally successful at reproducing the mass yields, $Y(A)$, but accurate calculations of $\overline{\operatorname{TKE}}(A)$ remain elusive. As discussed in these proceedings [1], density functional theory is being utilized to obtain the fragment excitation energy which could then be used in place of $\overline{\operatorname{TKE}}(A)$ to determine the early state of the fission fragments. These calculations, when more mature, might provide a reliable alternative means to obtain $\overline{\operatorname{TKE}}(A)$ when no data are available, as is often the case, especially for higher excitation energies in general and minor actinides in particular.

In standard FREYA [4], the initial nucleus of mass number $A_{0}$ divides into light and heavy fragments, with mass numbers $A_{L}$ and $A_{H}$ respectively. A tentative value of the total fragment kinetic energy, TKE, is given by $\overline{\operatorname{TKE}}\left(A_{H}\right)$ and the mean total excitation energy available for rotational and statistical excitation at scission is then $\bar{E}_{\mathrm{sc}}^{*}=Q-\overline{\mathrm{TKE}}$ where $Q=M_{0} c^{2}-M_{L} c^{2}-$ $M_{H} c^{2}$ is the $Q$-value for that particular split. The corresponding 'scission temperature' $T_{\mathrm{sc}}$ is obtained from $\bar{E}_{\mathrm{sc}}^{*}=a\left(A_{0}\right) T_{\mathrm{sc}}^{2}$ where the scale of the level density parameter $a(A)=A / e_{0}$, with $e_{0} \approx 10 \mathrm{MeV}$, is an adjustable parameter in FREYA.

The two nascent fission fragments are assumed to be formed with angular momenta perpendicular to the fission axis. In addition to their share of a possible overall rigid rotation

\footnotetext{
*e-mail: Vogt2@LLNL.gov

**e-mail: JRandrup@LBL.gov
} 
of the fissioning complex, the two angular momenta also acquire thermal fluctuations from the dinuclear wriggling and bending modes. The magnitude of these 'spin fluctuations' is governed by the 'spin temperature' $T_{S}=c_{S} T_{\text {sc }}$ which can be adjusted through the FREYA parameter $c_{S}$. (Thus the spin fluctuations vanish for $c_{S}=0$.)

After subtracting the resulting rotational energy of the two fragments, $E_{\mathrm{rot}}$, from $\bar{E}_{\mathrm{sc}}^{*}$ the remainder is the combined mean statistical excitation of the two fragments, $\bar{E}_{\mathrm{stat}}=\bar{E}_{\mathrm{sc}}^{*}-E_{\mathrm{rot}}$. A preliminary partition, $\bar{E}_{\text {stat }}=E_{L}^{*}+\hat{E}_{H}^{*}$, is made according to the heat capacities of the fragments, assumed to be proportional to the level density parameters, i.e. $\hat{E}_{L}^{*}: \hat{E}_{H}^{*}=a_{L}: a_{H}$. Because the observed neutron multiplicities for actinide nuclei at low energies suggest that the light fragments tend to be disproportionately excited, the light fragment is given a larger excitation energy by the FREYA parameter $x, \bar{E}_{L}=x \bar{E}_{L}, \bar{E}_{H}=E_{\text {stat }}-\bar{E}_{L}$, where $x>1$.

After the mean fragment excitation energies $\bar{E}_{i}$ have been assigned, FREYA considers thermal fluctuations in the statistical excitation. The mean fragment excitation is related to the fragment temperature $T_{i}$ by $\bar{E}_{i}=a_{i} T_{i}^{2}$ with the associated variance being $\sigma_{E_{i}}^{2}=2 \bar{E}_{i} T_{i}$. An energy fluctuation $\delta E_{i}^{*}$ is sampled from a truncated normal distribution of variance $2 c \bar{E}_{i} T_{i}$ and the excitations are adjusted accordingly, $E_{i}^{*}=\bar{E}_{i}+\delta E_{i}^{*}, i=L, H$. Energy is conserved by a compensating opposing fluctuation in TKE, TKE $=\overline{\mathrm{TKE}}-\delta E_{L}^{*}-\delta E_{H}^{*}$. The factor $c$ multiplying the variance is another adjustable parameter in FREYA with $c$ typically larger than unity. The parameter compensates for the truncation of the normal distribution due to energy conservation. We note that for spontaneous fission of some isotopes with low average neutron multiplicities but relatively broad multiplicity distributions $P(v), c$ can be as large as 3 [5]. Finally, to reproduce the average neutron multiplicity, $\bar{v}$, the overall average TKE may be adjusted by a fifth FREYA parameter, $d$ TKE.

The values of these five explicit parameters in the standard version of FREYA, $e_{0}, c_{S}, x$, $c$ and $d$ TKE, can be adjusted to data. Three are tightly correlated with specific observables, having little to no direct effect on others: The photon emission depends directly on $c_{S}$; the parameter $x$, governing statistical excitation energy sharing, determines both the neutron multiplicity as a function of fragment mass, $v(A)$, and the angular correlations between neutrons emitted during a fission event; and $c$ influences the neutron multiplicity distribution $P(v)$ and its moments. The only observable directly affected by $e_{0}$ is the prompt fission neutron spectrum (PFNS), though the other parameters also exhibit some influence over the PFNS. The parameter $d \mathrm{TKE}$, while related to the other parameters, serves primarily to ensure that $\bar{v}$ matches the evaluated value. Details about the parameter values and their correlations are given in Ref. [5].

After the fragments have been characterized, neutron and photon emission follows. The neutrons are evaporated isotropically in the frame of the emitting fragment, apart from a slight flattening due to the fragment rotation [6]. Their energy is sampled from a black-body energy spectrum, $d N_{n} / d E_{n} \sim E_{n} \exp \left(-E_{n} / T_{\max }\right)$, where $T_{\max }$ is the maximum possible temperature in the daughter nucleus. FREYA generally assumes that neutron evaporation continues until the nuclear excitation energy is below the neutron separation energy $S_{\mathrm{n}}$, so that neutron evaporation continues as long as energetically possible.

After neutron evaporation has ceased, the excited product nucleus emits photons. First, statistical photons are emitted isotropically with an energy distribution sampled from a blackbody spectrum modulated by a giant-dipole resonance form factor. When the nuclear excitation energy enters the regime of the tabulated decays from the RIPL-3 compilation [7], FREYA switches to a discrete cascade which is continued until the half-life exceeds a specified value, $t_{\max }$, based on the detector response time, or until the nucleus is in its ground state. The average photon energy and photon multiplicity depend on $t_{\max }$ as well as on the minimum photon energy measurable in the detectors, denoted in FREYA as $g_{\min }$. While the photon ob- 
servables depend on both $t_{\max }$ and $g_{\min }$, these quantities are not parameters but depend on specific experimental details.

It is clear that while FREYA has the five physics-based adjustable parameters described above, there are also many implicit inputs that rely on models and data, including fission barriers, nuclear masses, photon strength functions and the input yields themselves. For a study of the FREYA sensitivity to the input yield functions, see Ref. [8]. Because of its speed and adjustable formats for inputs, one of which is described in Sec. 2, FREYA can be used to test input yields and excitation energies calculated with models. Including data for delayed emission, FREYA can also be used to simulate cumulative yields in different time windows as well as provide a way to track the delayed emission in addition to prompt emission, as is outlined in Sec. 3.

\section{FREYA in $r$-process nucleosynthesis calculations}

While FREYA has been released for only a dozen isotopes, many more are required for calculating nuclear abundances in the $r$ process. See Ref. [9] for a more thorough discussion of the astrophysics processes. The discussion here will focus only on the role of FREYA in such calculations. As Ref. [9] made clear, fission fragments can be important contributors to the nucleosynthesis abundance patterns for neutron-rich astrophysical sites such as neutron star mergers. The neutrons emitted by the fission fragments may also be important, especially at later times, because they contribute to neutron capture,

In the $r$ process, fission can be initiated several ways: neutron-induced, beta-delayed, and spontaneous. Different fission processes are important at different time periods in the $r$ process. The different types of fission also occur at different compound nucleus excitation energies. To take these fission initiators and excitation energies into account, it is necessary to have more accurate calculations of the fission fragment mass distributions and the neutrons emitted during their de-excitation for models of astrophysical processes. Models are required because data for fission of high- $Z$ neuron-rich nuclei involved in fission recycling do not exist and are likely to remain unavailable. Heretofore, more naive treatments have been employed, including 50/50 splits and semi-empirical formulas. Previously, treating the yields and neutron emission from spontaneous, neutron-induced, and beta-delayed fission as distinct by taking into account their unique excitation energy dependence had not been done.

The work in Ref. [9] changed that. The 2016 version of the GEF code [10], which uses systematics in $Z, A$ and excitation energy of the compound nucleus based on data and models to calculate the fission fragment yields and excitation energies for neutron emission from the fragments, was employed. FREYA was also employed in the study to quantify the uncertainties in the resulting abundance pattern based on the de-excitation processes in the two codes. To do this, FREYA had to be modified to accept the GEF yields: $Y(A, Z), Y(A)$ and $Y$ (TKE).

Before proceeding, we investigated two test cases, ${ }^{252} \mathrm{Cm}(\mathrm{sf})$ and ${ }^{258} \mathrm{Pu}(\mathrm{sf})$, to see if FREYA could successfully incorporate the GEF yields and produce reasonable results for neutron-rich nuclei, far outside the regime where it had been previously applied. Results for the test cases are shown in Fig. 1.

The results in Fig. 1 showed that FREYA could take the GEF yields and faithfully reproduce them. It also shows that there are some differences in the fission product yields obtained with the two codes, due to the differences in the sharing of the excitation energy between the fragments. This difference is easier to see in the neutron multiplicity as a function of fragment mass. While $\bar{v}(A)$ from the two codes is in rather good agreement for the default parameter of $x$ used in the FREYA calculation of ${ }^{252} \mathrm{Cm}$ (sf) in the range $100<A<150$, there are discrepancies for the more asymmetric splits. This is not always the case, as seen 

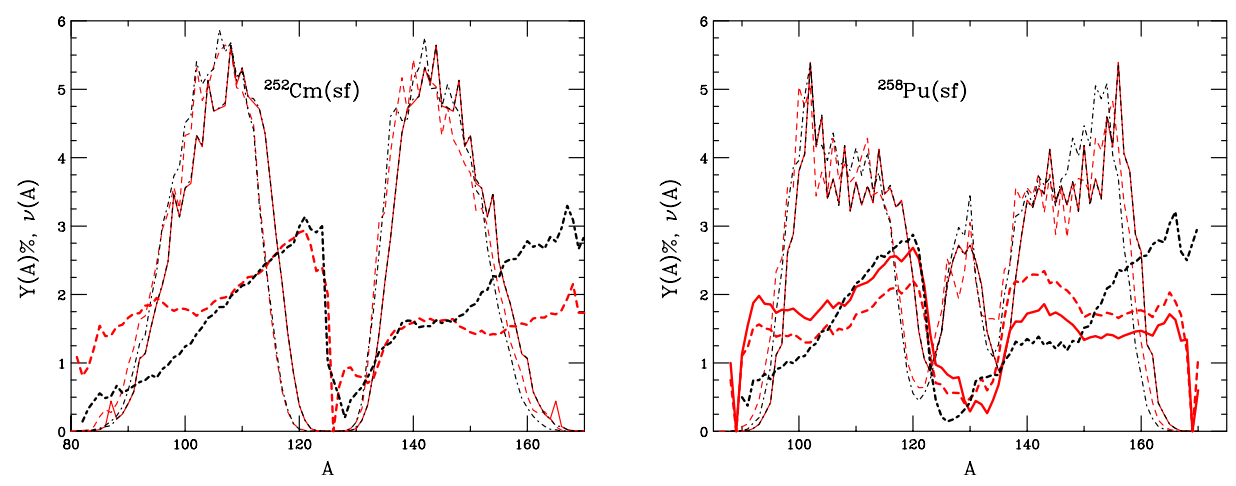

Figure 1. The fission fragment and fission product mass yields as well as the mean neutron multiplicity as functions of fission fragment mass number $A$ for ${ }^{252} \mathrm{Cm}$ (sf) (left) and ${ }^{258} \mathrm{Pu}$ (sf) (right). The thin solid red curves are the fission fragment yields from FREYA based on the GEF yields, underneath the red curves, in black. The thin dashed red curves are the FREYA fission product yields, after prompt neutron emission, while the thin black dot-dashed curve is the GEF result. The $Y(A)$ results are given in percent and are normalized to two fragments per fission, or $200 \%$. The thick curves show the mean neutron multiplicity as a function of the primary fission fragment mass. The red curves show the FREYA results for $\bar{v}(A)$, while the black curve shows the GEF results. Note that the calculations for ${ }^{258} \mathrm{Pu}(\mathrm{sf})$ (right-hand panel) show two results for FREYA. The dashed curve uses a default value for the excitation energy sharing parameter $x, x=1.15$, also used for the Cm calculation on the left, while the solid curve uses $x=1.4$, fixed to match the GEF value of the average neutron multiplicity from GEF. Note that the FREYA $Y(A)$ for fission product yields is obtained using $x=1.4$.

for ${ }^{258} \mathrm{Pu}(\mathrm{sf})$ where the value of $x$ used in FREYA had to be increased to 1.4 to reproduce the average neutron multiplicity $\bar{v}$ from GEF.

Because tuning parameters to reproduce the GEF results would provide little new information while giving no hint of uncertainties in the results from the two codes, we used the parameters for ${ }^{252} \mathrm{Cf}$ for spontaneous fission and ${ }^{239} \mathrm{Pu}$ for neutron-induced and beta-delayed fission. We found that FREYA generally resulted in higher neutron emission from fission than GEF, sometimes 2-3 more neutrons per fission. The difference is largest in the most neutronrich nuclei on the nuclear chart and comes mostly from the light fragment. This could be due to the fact that neutron emission from GEF is internally consistent, albeit with large uncertainties, while although FREYA starts from the same yields it handles the excitation energy sharing differently than does GEF. These differences can result in 10-20\% relative changes in the abundance pattern between FREYA and GEF. See Ref. [9] for full details.

\section{FREYA for Cumulative Yields}

The current version of FREYA simulates spontaneous or induced fission from the precompound stage through prompt neutron and photon emission to the resulting product nuclei. These are generally beta unstable and, consequently, on a longer time scale, they will undergo a sequence of beta decays accompanied by photon radiation. We plan to incorporate these delayed processes into FREYA to be able to simulate the decays leading to cumulative fission yields. With this information incorporated, we can compare to fission product yield measurements at different time scales, from seconds to minutes to days. Because FREYA simulates complete events, it is also possible to follow the trajectories of the delayed particles that are emitted, including the neutrons, photons, electrons and antineutrinos. (Note that for the $r$ - 
process application described in Sec. 2, delayed emission from fission products was treated in the nucleosynthesis network calculation using the decay data described in Ref. [9].

If the needed information about the delayed processes is available, then the effort required to incorporate these processes into the existing version of FREYA should be relatively manageable through a new subroutine called after the prompt processes have ceased. This routine will follow the delayed decay processes in a Monte-Carlo manner and, for each product nucleus. it will provide both the resulting beta-stable fission fragment daughter and the kinematic information about the emitted photons, and leptons (electrons and antineutrinos). It should be relatively straightforward to construct and implement such a subroutine without slowing FREYA down by any appreciable degree.

The FIER code package [11] contains essentially all the known information on the betadelayed decay processes. In FIER, the available information is tabulated in two files: one containing the half-lives and associated branching ratios and the other the beta-decay end point energies and associated intensities. The $N, Z$ domain covered by these data files are illustrated in black and red respectively on the left-hand side of Fig. 2. However, some portion of the beta-decay data is not available and therefore the incorporation of these processes must rely on some modeling. We do note that many of the fission products from spontaneous fission of ${ }^{252} \mathrm{Cf}$ do have data available so that modeling is not required for every possible fission product. This does, of course, depend on the fissioning isotope as well as its excitation energy.
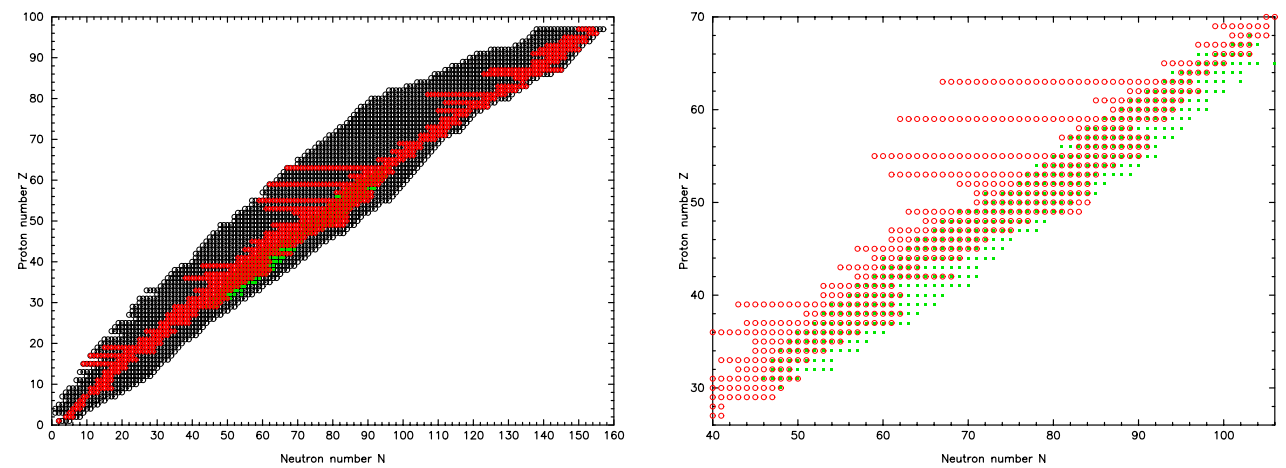

Figure 2. (Left) The $(N, Z)$ domains included in the FIER data tables. The dark bins show the nuclides with branching ratios and half-lives included, while the overlaid red bins show those with information on beta-decays. Clearly there is beta-decay information available for far fewer nuclides. The $(N, Z)$ distribution of the fission products from spontaneous fission of ${ }^{252} \mathrm{Cf}(\mathrm{sf})$ is shown by the green bins. It is apparent that most but not all of the nuclei of interest are contained in the FIER tables. (Right) Magnified views of the relevant parts of the $(N, Z)$ plane for ${ }^{252} \mathrm{Cf}(\mathrm{sf})$. The half-life information is omitted because all fission fragments of interest are included. Note that the green symbols show the location of fission products from one million spontaneous-fission events.

Because accurate modeling of the beta-delayed decay processes is difficult, it is unrealistic to imagine that a fully satisfactory theoretical model of beta-delayed decay chains will be available in the near future. However, the state of the art is steadily improving.

It is therefore preferable to start with a relatively simple form of modeling while ensuring that the augmented version of FREYA is structured so that model replacements can readily be made. It will then be relatively easy to maintain a state-of-the-art treatment while also allowing comparisons between alternative decay models. 


\section{Summary}

FREYA is a robust, fast, flexible tool for studying complete fission events. While it currently handles only prompt emission, allowing access only to the independent fission product yields, it can be extended to include delayed emission by incorporating the FIER data, giving access to the cumulative fission product yields as well.

\section{Acknowledgments}

We thank R. Surman and N. Vassh for collaboration on the $r$-process calculations. The work of R.V. was performed under the auspices of the U.S. Department of Energy by Lawrence Livermore National Laboratory under Contract DE-AC52-07NA27344. The work of J.R. was performed under the auspices of the U.S. Department of Energy by Lawrence Berkeley National Laboratory under Contract DE-AC02-05CH11231. This work was supported by the Office of Defense Nuclear Nonproliferation Research \& Development (DNN R\&D), National Nuclear Security Administration, U.S. Department of Energy.

\section{References}

[1] N. Schunck, these proceedings.

[2] M. Verriere, these proceedings.

[3] I. Abdurrahman, these proceedings.

[4] J. M. Verbeke, J. Randrup and R. Vogt, Comp. Phys. Comm. 222, 263 (2018).

[5] J. T. Van Dyke, L. A. Bernstein and R. Vogt, Nucl. Instrum. Meth. A 922, 36 (2019).

[6] J. Randrup and R. Vogt, Phys. Rev. C 89, 044601 (2014).

[7] R. Capote et al., Nucl. Data Sheets 110, 3107 (2009).

[8] J. Randrup, P. Talou and R. Vogt, Phys. Rev. C 99, 054619 (2019).

[9] N. Vassh et al., J. Phys. G 46, 065202 (2019).

[10] K.-H. Schmidt, B. Jurado, C. Amouroux and C. Schmitt, Nucl. Data Sheets 131, 107 (2016).

[11] E. Matthews et al., Nucl. Istrum. Meth. A 891, 111 (2018). 